\title{
THE PISTON MODEL OF CORONAL TRANSIENTS
}

\author{
WEN-RUI HU and ZE-MEI TANG \\ Institute of Mechanics, Academic Sinica, Beijing. China
}

(Received 18 November, 1983)

\begin{abstract}
In the present paper, the piston model of the coronal transient (see Hu, 1983a, b is discssed in detail, and the quantitative results of unsteady gasdynamics are applied to the coronal transient processes. The piston model explains the major features of the transient observations, such as. the density profile, the geometric configuration, the kinetic process and the classifications of the coronal transient. Based on the idea of piston model, the bright feature and the dark feature of the transient are the gasdynamical response of the dense plasma ejecting into the corona, and associate with the compressed and rarefied flows, respectively. The quantitative results show that the density increment in the compressed region and the density decrement in the rarefied region are one order of magnitude aarger and smalsar, respectively, to the density in the quiet corona, it agrees quantitatively with the observations, and both the bright feature and dark feature are explained at the same time.
\end{abstract}

\section{Introduction}

The coronal transients are important events of solar activities which have enormous amounts of energy associated with mass ejections. The observations show that the features of coronal transients not only have a variety of configurations, but also include the kinetic and dynamical processes. As a sort of newly discovered solar activity with large amount of mass ejection, it absorbs extensive interest in recent years. There has been much discussion about the observation features and several theoretical models are suggested (see, for example, the reviews by Rust et al., 1979; MacQueen, 1980, or Dryer, 1982).

Statistical analyses of the observations show the correlations between the coronal transient and other solar activities, such as the eruptive prominence and solar flare. During the Skylab period, more than $70 \%$ of the coronal transients are associated with the events of eruptive prominences or filament disappearances which may be with or without the solar flare (Munro et al., 1979). A great amount of gas is often ejected through the corona into the interplanetary space in the process of solar flare, such as the manifestion of type II burst. The eruptive prominence is also an ejection of dense plasma in the corona. In the processes of other solar activities, there generally are ejections of dense plasma into the corona. Therefore, the ejection of dense plasma produced by the solar activities may be the driven source or the triggering factor of the coronal transient.

The coronagraph measurements in space give the bright feature of coronal transient in the range of 2-6 solar radii. In comparison with the background density in quiet corona, the bright region associates with higher density of plasma. On the other hand, the observations made with a ground-based coronameter supply informations in the lower corona, where the dark region of coronal 


\section{Quiet Corona}

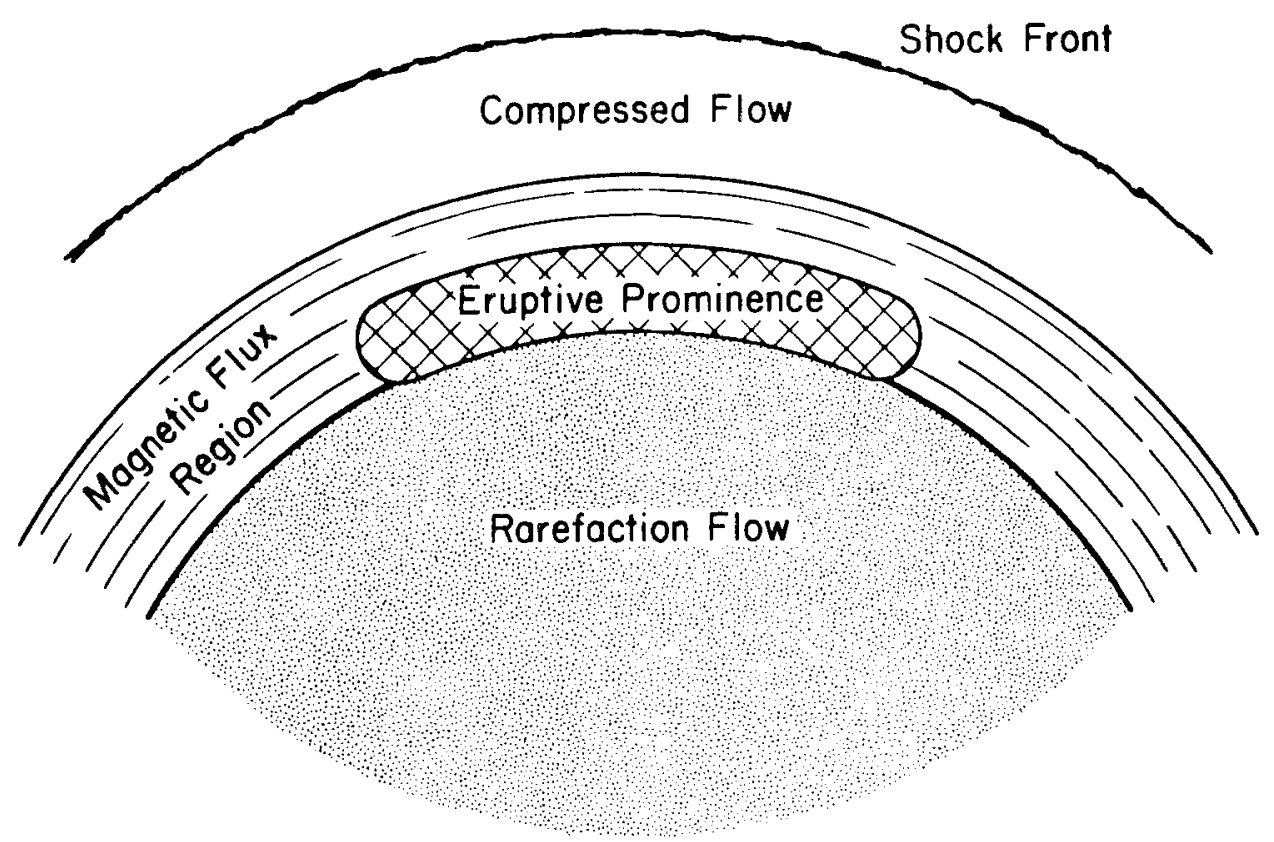

Fig. 1. The physical picture of the blob transient, where the configuration is axisymmetric.

transient associates with a lower plasma density. It is identified that the prominence locates ahead of the dark region in an event of transient associated with eruptive prominence. Therefore, a theoretical model of coronal transient should explain the complete process including both the bright and dark features.

A piston model has been suggested to explain the coronal transient associated with eruptive prominence, as shown in Figure 1 ( Hu, 1983a, b). As the dense plasma ejecting into the corona with closed magnetic field, the magnetic field will be pushed ahead the dense plasma to form a magnetic compressed region, such as a loop, arcade or filled bottle. If the dense plasma moves outward faster, so does the compressed magnetic field region ahead of the moving plasma, and the foot of the compressed magnetic field will expand slowly. If we consider only the compressed magnetic region, for example, a loop, it seems that there is a similarity between the piston model and the loop model. However, some of the loop models consider only the motion of the loop itself (for examples, Mouschovias and Poland, 1978; Anzer, 1978; Liu, 1983), while others analyze the interaction between the loop and the environment outside it, but the gas-dynamical effects which will be induced in the corona by the moving loop are not concerned (for examples, Pneuman, 1980; Yeh and Dryer, 1981; Yeh, 1982; Anzer and Pneuman, 1982). 
Based on the idea of the piston problem in gas dynamics, a compressed flow with higher density between the piston and the shock front and a rarefunction flow with lower density will be formed ahead of and behind the piston, respectively. Therefore, as the ejecting dense plasma and compressed magnetic region are considered as the moving piston, the piston model may explain the bright features and the dark features of transient at the same time. On the other hand, the results of numerical computations of unsteady and two-dimensional models show a lot of gas-dynamical processes of coronal transients (see, for example. Nakagawa et al., 1978, 1981; Steinolfson and Nakagawa, 1977; Wu et al., 1978; Dryer et al., 1979).. These results depend on the adoptions of the initial and boundary conditions, and a pulsation of a thermodynamical quantity or the magnetic field is often assumed at the low coronal boundary as the driven course. This assumption is different from one in the present model, and the mechanism will be different.

From the viewpoint of gas dynamics, the piston model should analyze the energy conversions between the gravitational potential, kinetic and internal energies, it is different from the usual piston problem in gas dynamics where the applied gravity is excluded. The influence of applied gravity on the piston problem is dicussed elsewhere (Hu, 1984; Tang, 1984a,b), and may be applied to the dynamical process of coronal transients. We suggested the piston model of coronal transient by a simple analysis approach (Hu, 1983a, b). However, in the present paper we deal - more quantitatively - with the model of spherical piston, which includes the curvature of the configuration. The quantitative results are easy to compare with the observations.

In the next section, the mathematical descriptions of the spherical piston model, including the basic equations and the boundary conditions, are given. In Section 3, the qualitative results of the piston model are discussed. The theoretical model is compared with the observations in Section 4, and there is conclusion in the last section.

\section{The Piston Model}

The observations of the coronal transient are the projects of the three-dimensional configurations in the plane perpendicular to the direction of view. We discuss a transient of cloud or blob configuration, which is driven by the piston consisted of a dense plasma and a compressed magnetic region. The spherical coordinate is convenient to describe the processes near the top of these transients, and is shown as in Figure 2. In this case, the gas-dynamical equations may be written as

$$
\begin{aligned}
& \frac{\partial \rho}{\partial t}+\rho \frac{\partial v}{\partial r}+v \frac{\partial \rho}{\partial r}+\frac{2 \rho v}{r}=0, \\
& \rho\left(\frac{\partial v}{\partial t}+v \frac{\partial v}{\partial r}\right)=-\frac{\partial p}{\partial r}+\rho \frac{G M}{r^{2}}, \\
& \frac{\partial}{\partial t}\left(\frac{p}{\rho^{\gamma}}\right)+v \frac{\partial}{\partial r}\left(\frac{p}{\rho^{\gamma}}\right)=0,
\end{aligned}
$$




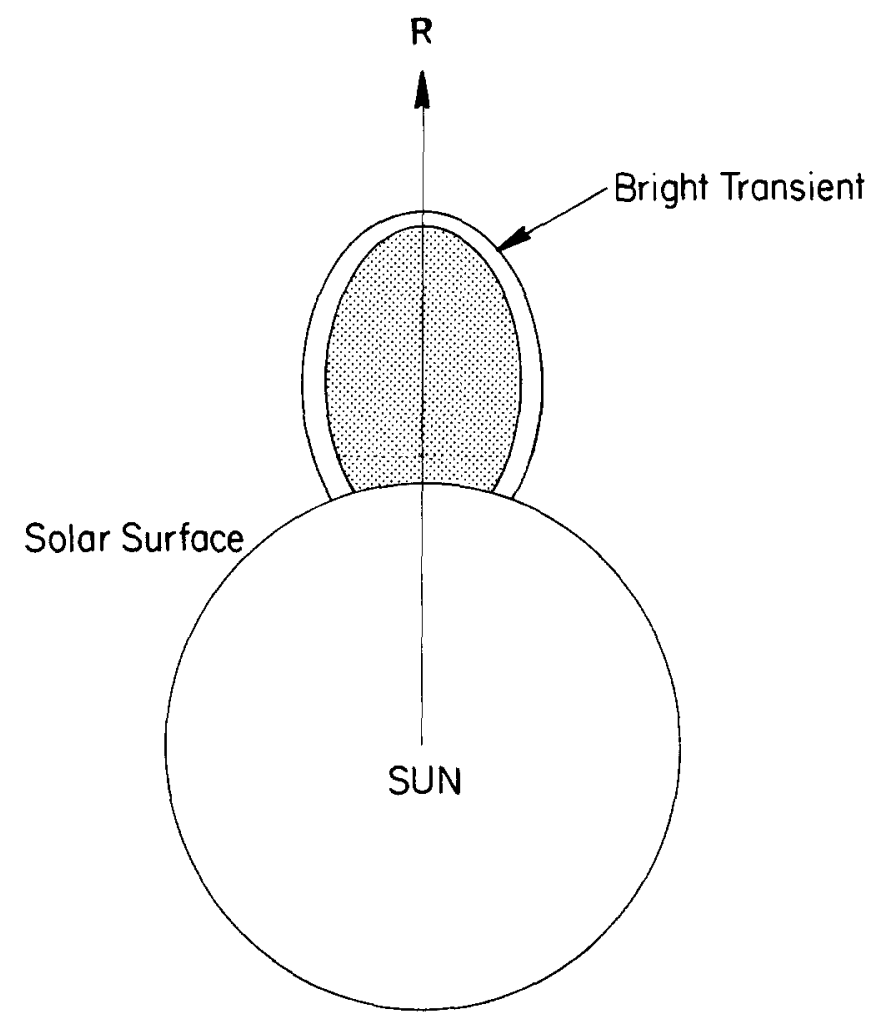

Fig. 2. The spherical coordinates are used in the present paper.

where $p, \rho$, and $v$ are the pressure, the density and the radial component velocity of plasma, respectively, and $G$ is the gravitational constant; $M$, the mass of the Sun; $\gamma$, the polytropic index; $t$, the time and $r$, the radial coordinate in the spherical coordinates. If we introduce the sonic velocity

$$
a=\left(\frac{\gamma p}{\rho}\right)^{1 / 2}
$$

Equations (2.1)-(2.3) are reduced into

$$
\begin{aligned}
& \frac{\partial v}{\partial t}+v \frac{\partial v}{\partial r}+\frac{2 a}{\gamma-1} \frac{\partial a}{\partial r}=-\frac{G M}{r^{2}}, \\
& \frac{\partial a}{\partial t}+v \frac{\partial a}{\partial r}+\frac{(\gamma-1) a}{2}\left(\frac{\partial v}{\partial r}+\frac{2 v}{r}\right)=0 .
\end{aligned}
$$

If we introduce the nondimensional quantities

$$
R=\frac{r}{r_{0}}, \quad \tau=\frac{t}{t_{0}}, \quad V=\frac{v}{r_{0} / t_{0}}, \quad A=\frac{a}{r_{0} / t_{0}},
$$


and nondimensional gravitational velocity

$$
V_{q}=\frac{t_{0}}{r_{0}}\left(\frac{G M}{r_{0}}\right)^{1 / 2}
$$

the nondimensional equations of (2.5) and (2.6) are

$$
\begin{aligned}
& \frac{\partial V}{\partial \tau}+V \frac{\partial V}{\partial R}+\frac{2 A}{\gamma-1} \frac{\partial A}{\partial R}=-\left(\frac{V_{g}}{R}\right)^{2}, \\
& \frac{\partial A}{\partial \tau}+V \frac{\partial A}{\partial R}+\frac{(\gamma-1) A}{2}\left(\frac{\partial V}{\partial R}+\frac{2 V}{R}\right)=0 .
\end{aligned}
$$

Equations (2.9) and (2.10) are reduced to the equations of usual plane piston problem in the gas dynamics if the right-hand term, which gives the influence of the applied gravity, and the third term, in the left-hand side, which gives the curvature influence of spherical coordinates, in Equation (2.9) are zero. It will be seen that the gravitational term has great influence on the distribution of flow field, and the curvature term will not change the physical picture but changes the quantitative results in the process. In the following discussion, we adopt the typical parameter $r_{0}=1 r_{\odot}$ and $t_{0}=1 \mathrm{hr}$, and the typical velocity $r_{0} / t_{0} \simeq 195.5 \mathrm{~km} \mathrm{~s}^{-1}$.

We need two boundary conditions for Equations (2.9) and (2.10). One of the conditions is the piston velocity, which may be given typically as the observed velocity of the eruptive prominence. We adopt the nondimensional piston velocity as

$$
V_{p}= \begin{cases}n(0.465 \tau-0.46), & 1 \leqslant \tau \leqslant 2.31, \\ V_{p}(\tau=2.31), & 2.31 \leqslant \tau,\end{cases}
$$

where the subscription $p$ denotes the value at piston, $n$ is a constant and is adopted as 1 and 2. Another condition is the match condition between the flowing region and the quiet corona, where the static equilibrium relationship is satisfied - i.e.,

$$
\frac{\mathrm{d} p}{\mathrm{~d} r}=-\rho \frac{G M}{r^{2}}
$$

There is a shock wave in front of the compressed flow; and the jump conditions across the shock front are

$$
\begin{aligned}
& V_{s}=\frac{2}{\gamma+1} \frac{\theta^{2}-A_{c}^{2}}{\theta}, \\
& P_{s}=P_{c}+\frac{2 \Theta_{c}}{\gamma+1}\left(\theta^{2}-A_{c}^{2}\right), \\
& \Theta_{s}=\frac{\Theta_{c}}{\frac{\gamma-1}{\gamma+1}+\frac{2}{\gamma+1}\left(\frac{A_{c}}{\theta}\right)^{2}},
\end{aligned}
$$


where the subscriptions $s$ and $c$ denote the values just behind and ahead of the shock front in the compressed flow region and in the quiet corona, respectively, $\theta$ is the propagation velocity of shock wave divided by $x_{0} / t_{0}$ and nondimensional pressure and density are defined as

$$
P=\frac{p}{\rho_{0}\left(x_{0} / t_{0}\right)^{2}}, \quad \Theta=\frac{\rho}{\rho_{0}}, \quad \Theta_{c}=\frac{\rho_{c}}{\rho_{0}} .
$$

Shock conditions (2.14) and (2.15) may be reduced to

$$
A_{s}=\theta\left[\frac{\gamma-1}{\gamma+1}+\frac{2}{\gamma+1}\left(\frac{A_{c}}{\theta}\right)^{2}\right]^{1 / 2}\left\{\left(\frac{A_{c}}{\theta}\right)^{2}+\frac{2 \gamma}{\gamma+1}\left[1-\left(\frac{A_{c}}{\theta}\right)^{2}\right]\right\}^{1 / 2} .
$$

For the rarefaction flow behind the piston, we should give the boundary condition at the interface between the rarefraction flow and the quiet corona, this condition is

$$
\begin{aligned}
& V_{r}=0, \\
& A_{r}=A,
\end{aligned}
$$

where the subscript $r$ denotes the value in the rarefaction region just behind the interface. The quantity $A_{c}$ in Equation (2.13), (2.17), and (2.19) will satisfy the equilibrium relationship (2.12), and is determined after the polytropic index $\gamma$ is given.

In this present paper, we consider the coronal gas is in the polytropic state. The observations show that the temperature of the coronal gas decreases more slowly than it would for the adiabatic process (see, for example, Allen, 1973). Therefore, we adopt the polytropic index $\gamma=\frac{4}{3}$. Furthermore, the initial condition of moving piston is given as

$$
R_{0}=1.2, \text { when } \tau_{0}=1 \text {. }
$$

where the subscription 0 denotes the initial value.

By using these conditions, Equations (2.9) and (2.10) may be solved. The characteristic method is used for the piston problem. As the boundary conditions are given at two different surfaces, that is, one of which is the surface of piston trajectory and the other is the intersurface between the rarefaction flow or shock front and the quiet corona, the computational approach will be more complicate than one in the previous paper (Hu, 1983a). The interactive process of computations should be used to match the boundary conditions at different surfaces (see, for example, Tang, 1974b). The solutions of this piston problem will be given in the next section.

\section{The Flow Field}

The dynamical processes of the coronal transient include three typical major flow regions - that is, the compression flow region, the rarefaction flow region and the 


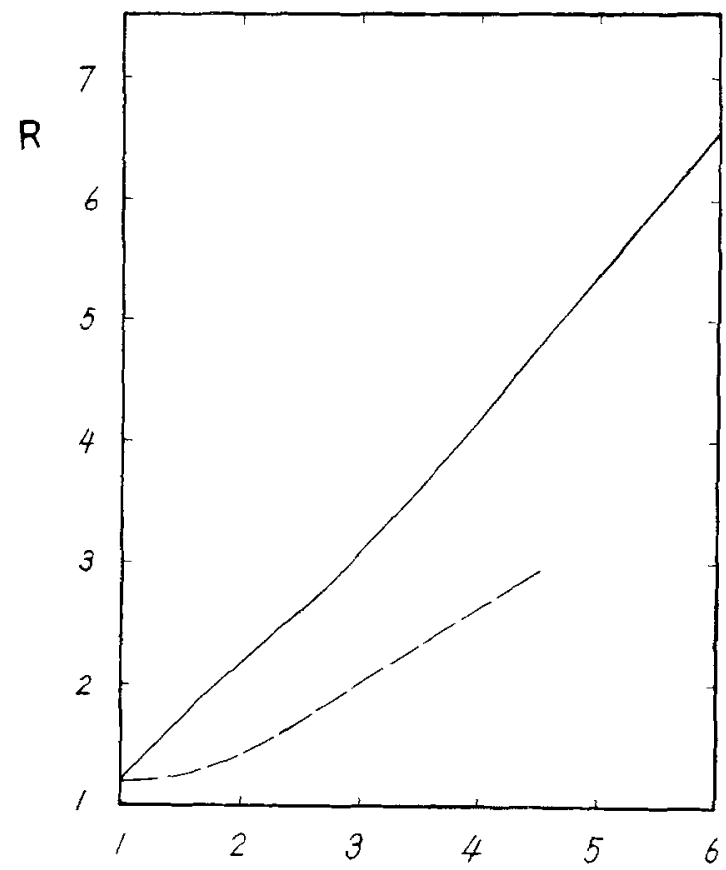

Fig. 3. The trajectories of piston and shock front expressed by the broken and full lines for $n=1$, respectively.

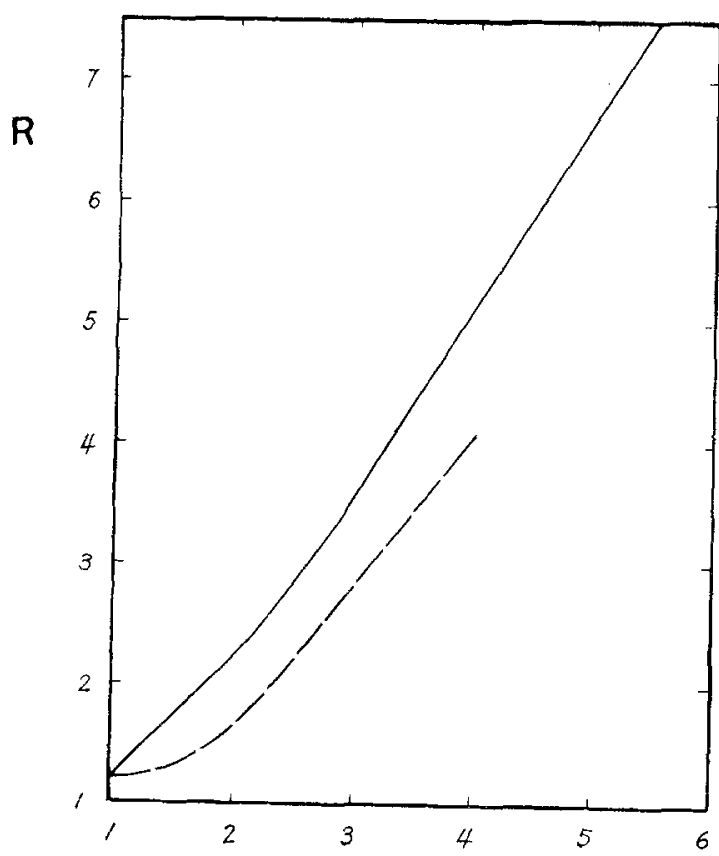

Fig. 4. The trajectories of piston and shock front expressed, respectively, by the broken and full lines $n=2$. 
region of piston which consists of the ejected dense plasma and the compressed magnetic region, as shown in Figure 1 . We describe the results first for the evolutionary changes in the compression flow region, followed by the one in the rarefraction region. The former corresponds to the bright features of the coronal transient, and the latter associates with the dark features of transient.

\subsection{COMPRESSION FLOW}

The compression flow region is between the piston and the shock wave. Figures 3 and 4 show the positions of the shock fronts for two case corresponding, respectively, to $n=1$ and 2 in Equation (2.11). In both cases, the velocity of piston is given by (2.11), and $v_{p}(2.31)=118.7 \mathrm{~km} \mathrm{~s}^{-1}$ for $n=1$ as shown in Figure 3 and $v_{p}(2.32)=237.4 \mathrm{~km} \mathrm{~s}^{-1}$ for $n=2$ as shown in Figure 4 . The distribution of piston velocity given here is typically similar to the one of eruptive prominence velocity. It can be seen that the shock front is only accelerated slightly, especially after a short beginning period, and the larger the speed, the narrower the distance between the piston and the shock front. The position of the shock front and the strength of the

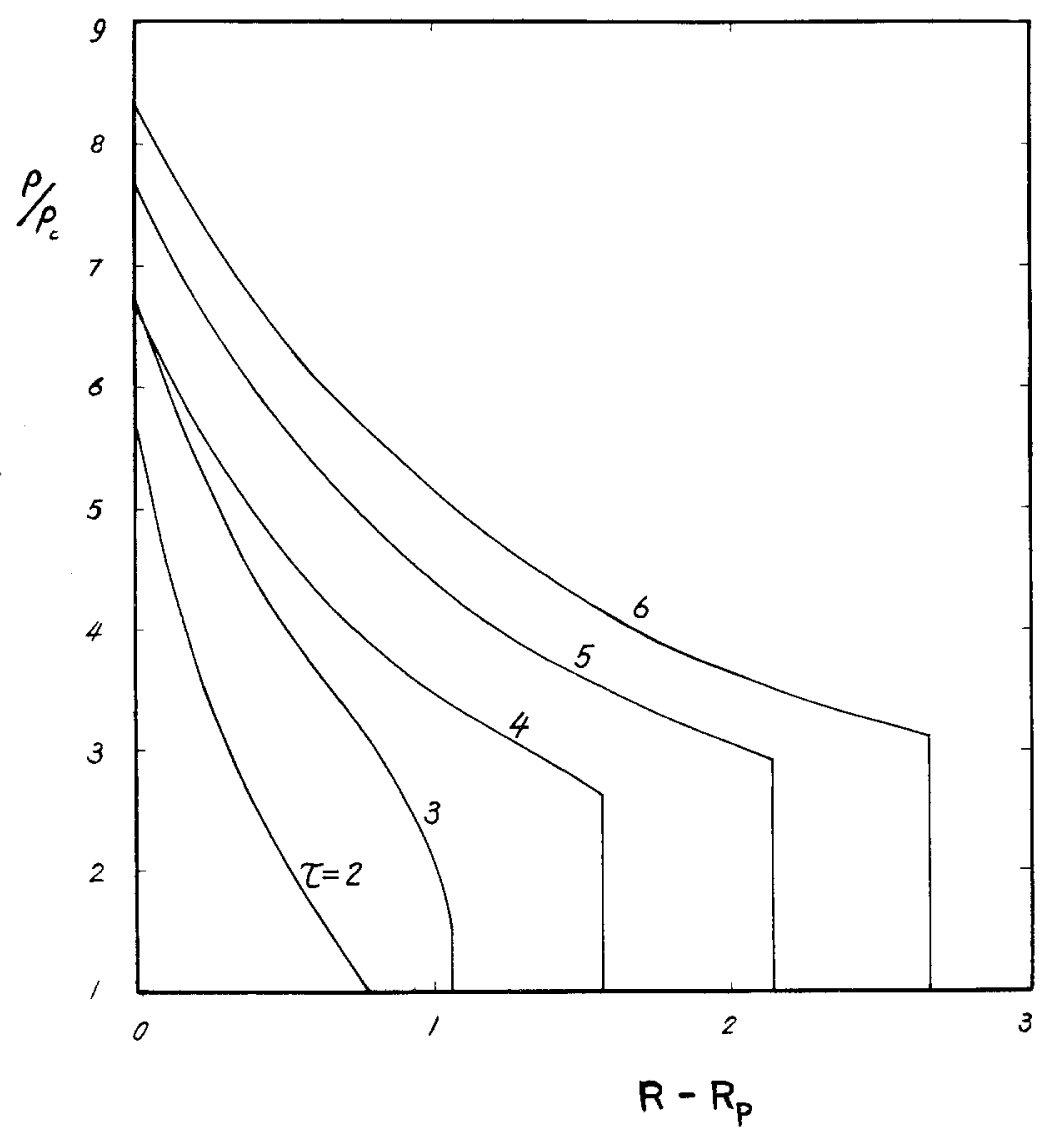

Fig. 5. The evolutions of plasma density profiles in the compression flow region for $n=1$. 
shock wave are determined by the velocity distribution of piston and the state of the quiet corona if the applied gravity is given. As the piston is accelerated slightly, so does the shock front. Furthermore, there is stronger compression in the case of higher piston velocity, and then, the distance between the piston and the shock front will be narrower.

The evolutions of density profiles have major importance in the transient processes. Figures 5 and 6 give the density profiles for the cases $n=1$ and 2 , respectively, where the ordinate denotes the nondimensional density $\Theta / \Theta_{c}$ which is the ratio of density $\Theta$ and the reference density $\Theta_{c}(\tau)$ at quiet corona just ahead of the shock front at time $\tau$, and the abscissa denotes the distance relative to the moving piston $R_{p}(R)$. The density increment consists of two parts, one partis the shock jump from the state of quiet corona just ahead of the shock to the compression flow region just behind the shock front, another part is produced by the compression effects and the influence of applied gravity in the compression flow region. Both figures show that the shock strength increases gradually from zero at the beginning, the larger the piston velocity, the greater the density increment by the shock jump. As the compression flow region is narrower in the case of larger piston velocity, the density increment in the compression flow region is a small amount for the case of great compression in comparison with the slight compression case, as shown clearly in Figures 5 and 6. However, the net density increment including the contributions of shock jump and the contribution in the

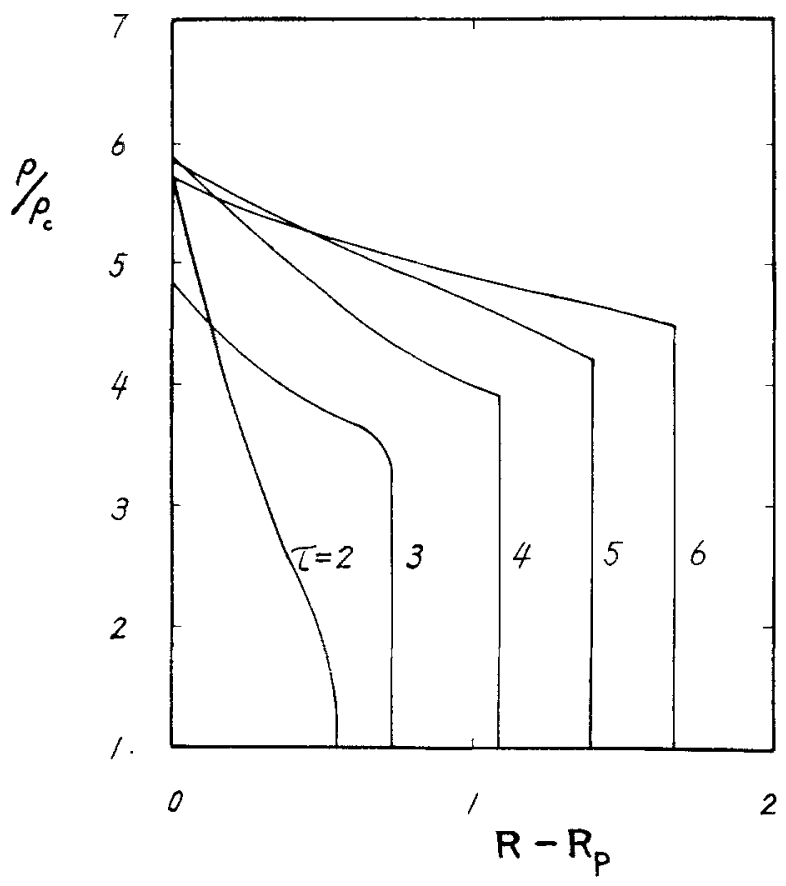

Fig. 6. The evolutions of plasma density profiles in the compression flow region for $n=2$. 
compression flow region is one order of magnitude larger than the density in the quiet corona, and the ratio of density increment by shock jump and by the compression flow region is increasing with the shock strength. These conclusions are easy to be understood after the applied gravity is included in the gas-dynamical processes (Tang, 1984b).

Figures 7 and 8 are the velocity profiles in the compression flow region. In the initial period, the velocity of plasma is decreasing with the distance $R-R_{p}$ increasing, but afterwards, the velocity tends to increase, especially in Figure 7. To overcome the applied gravity, there need the dissipations of some energies, for examples, the kinetic or internal energy of plasma. In consideration of Figures 5 and 6 , the internal energy of plasma dissipates in the compression flow region.

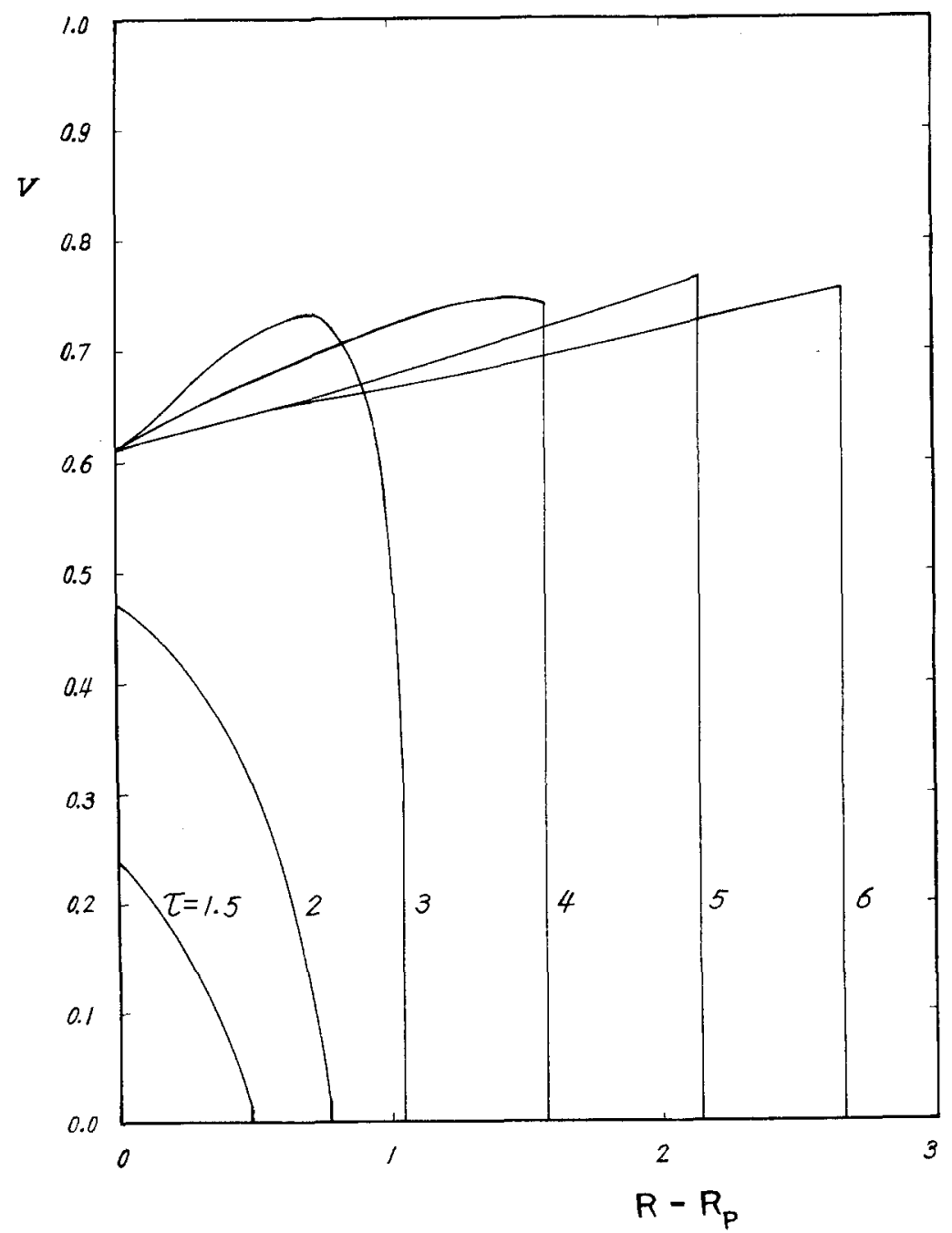

Fig. 7. The evolutions of plasma velocity profiles in the compression flow region for $n=1$. 


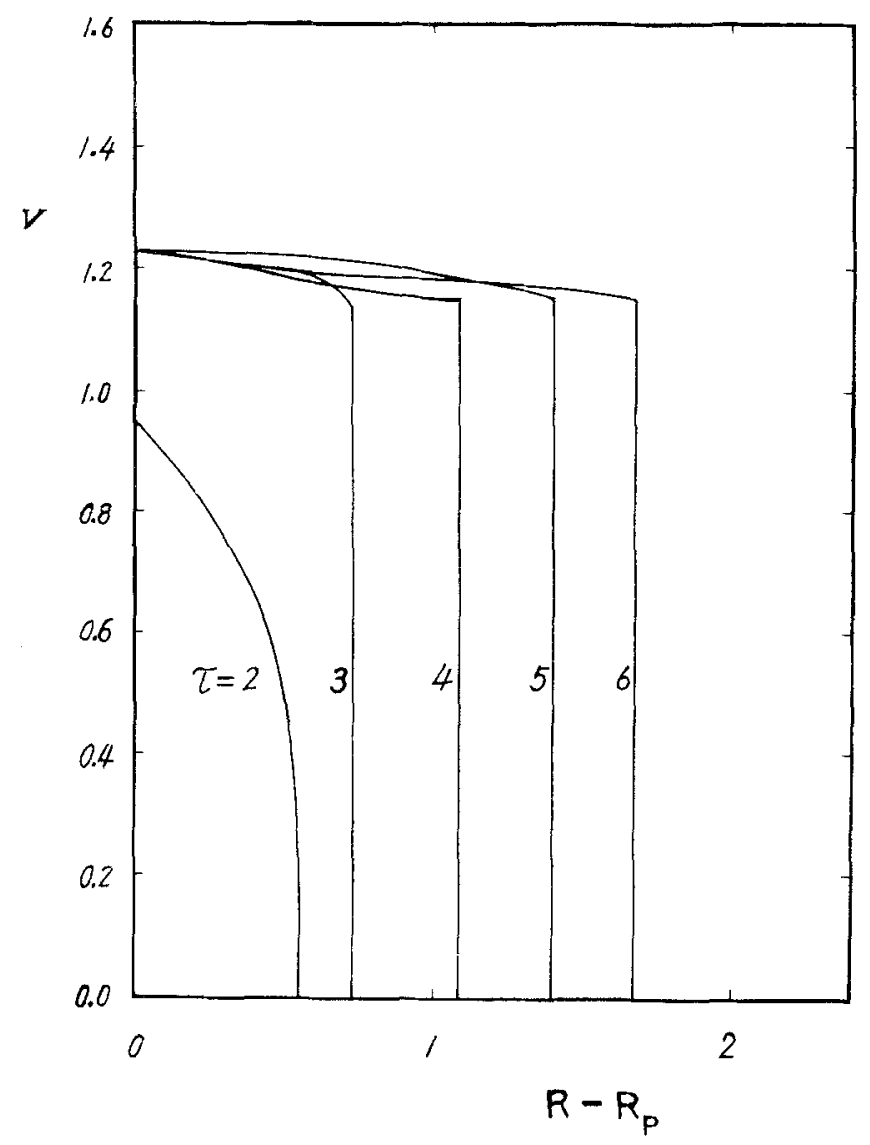

Fig. 8. The evolutions of plasma velocity profiles in the compression flow region for $n=2$.

Therefore, the internal and kinetic energies are dissipated to overcome the applied gravity in the initial period, and the internal energy is majorly dissipated to overcome the gravity and, sometimes, to accelerate the plasma in the later period. Comparing the results in Figures 7 and 8, we see that the larger the piston velocity, the greater the plasma velocity in the compression flow region. However, the acceleration of plasma shows more clearly in Figure 7, because the compression region is wider. As in the usual piston problem, the velocity of shock front is supersonic as shown in Figures 3 and 4, but the plasma velocity in the compression flow region is subsonic in the case of subsonic piston velocity.

\subsection{RaReFaction FLow}

The rarefaction flow is in the region between the piston and the weak discontinuous surface which seperates the rarefaction flow and the quiet corona behind the piston, and is determined by the velocity distribution at the piston and the condition at the intersurface of weak discontinuity as given in (2.18) and (2.19). However, the rarefaction flow will be stop above the solar surface, for example, at 


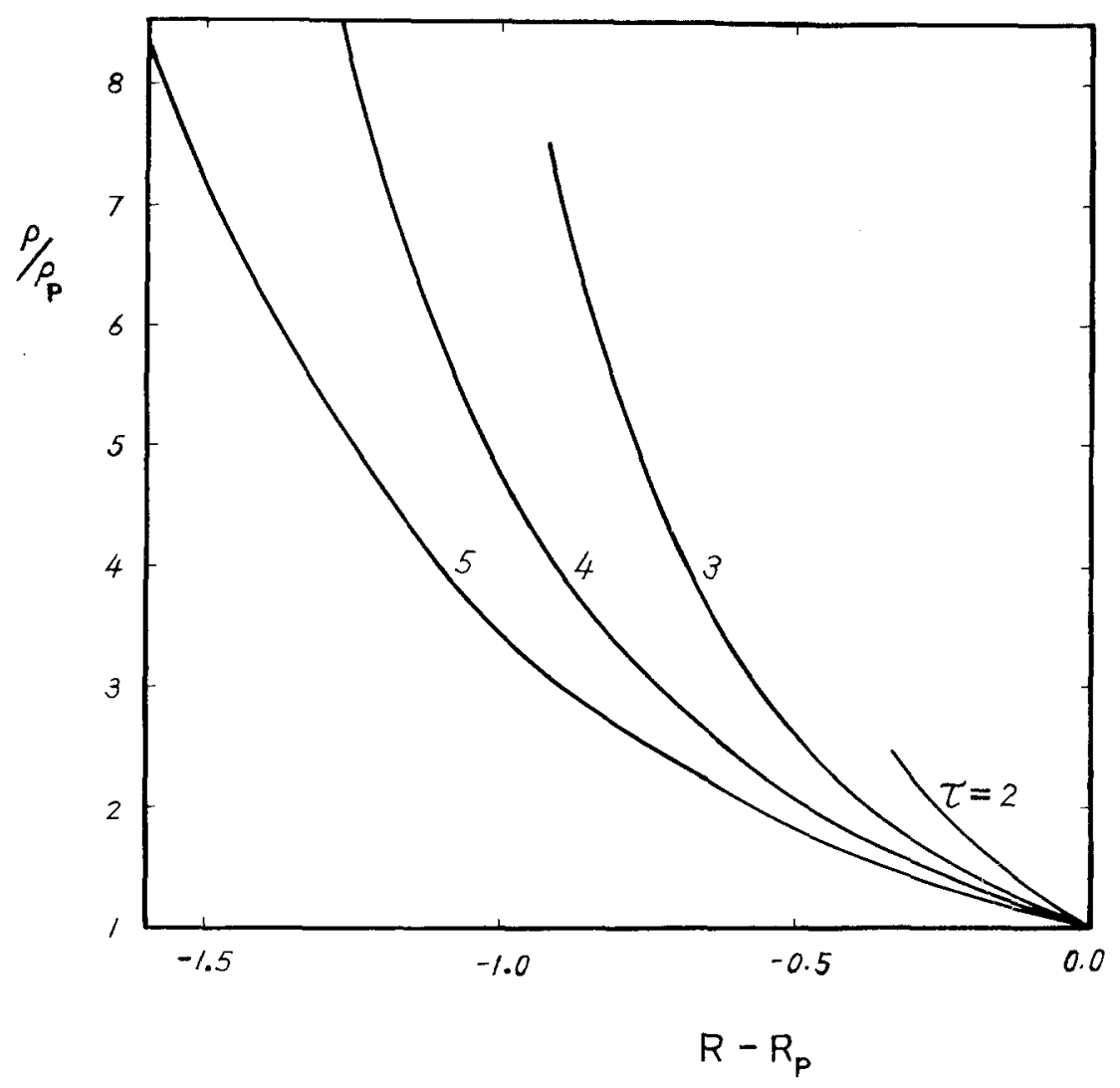

Fig. 9. The evolutions of plasma density profiles in the rarefaction flow region for $n=1$.

$R=R_{*}>1$. In addition to conditions (2.18) and (2.19) in the range $R>R_{*}$, and is adopted as

$$
A=A_{c}(R=1.1)-0.0367(\tau-1.0), \quad R=R_{*}=1.1,
$$

which agrees with the condition given by (2.18) and (2.19) without the truncation. Let us now discuss the rarefaction flow for the case $n=1$, and the result of rarefaction flow for $n=2$ are similar.

The evolution of density profiles shown in Figure 9, where the nondimensional density $\Theta / \Theta_{p}$ is the ratio of density $\Theta$ and the reference density $\Theta_{p}(\tau)$ at the piston at time $\tau$. It can be seen that the plasma density decreases from $R=R_{*}$ to the piston, and a lower density region exists obviously behind the piston. This region will be expanded with the piston moving outward, and the density decrement will be larger and larger.

The evolution of velocity profiles in the rarefaction region are shown in Figure 10. The velocity decreases as the distance $\left|R-R_{p}\right|$ increases. The tendency of velocity varieties is similar to the usual rarefaction flow in the gas dynamics. The applications of these results may explain the major feature of the coronal transient. 


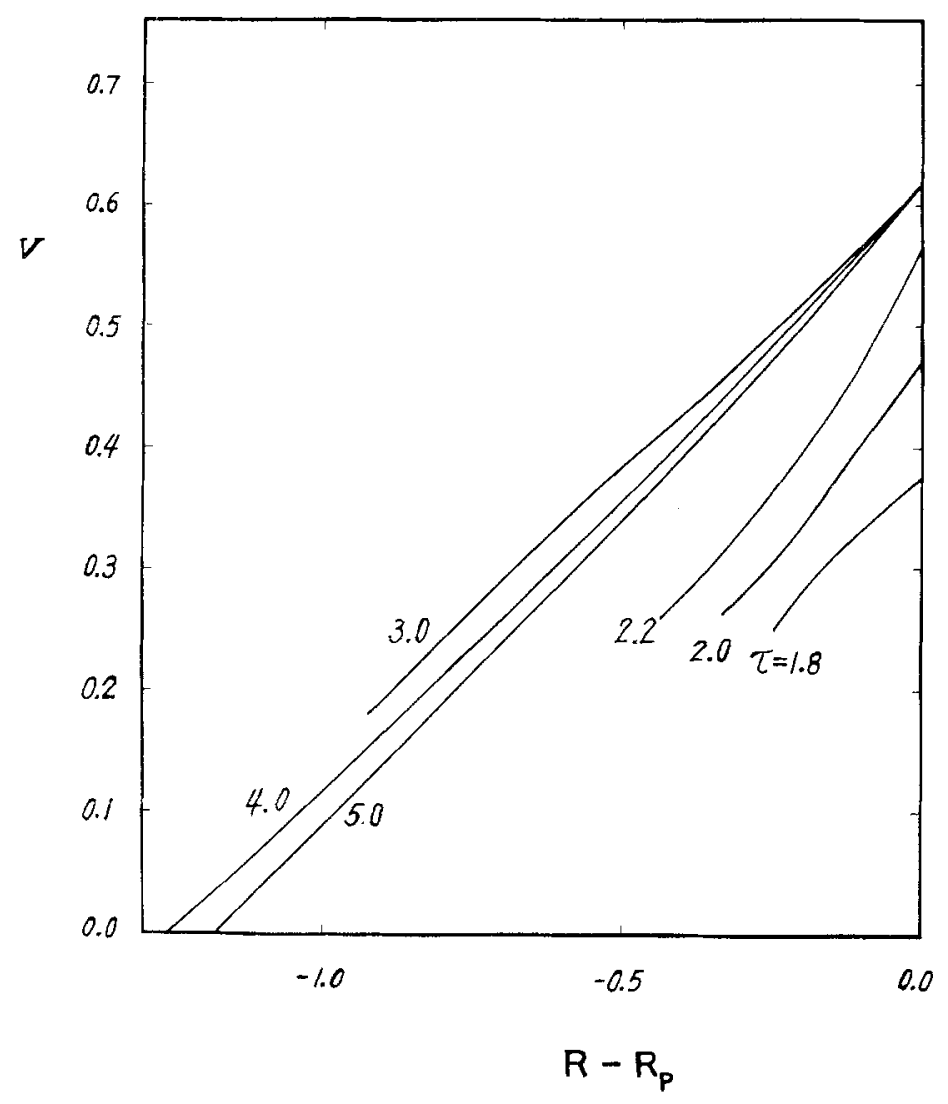

Fig. 10. The evolutions of plasma velocity profiles in the rarefaction flow region for $n=2$.

\section{Comparison with Observations}

The coronal transient represents a manifestation of solar activity involving a large amount of energy; and the observations of transient are concerned with such processes as the geometry, the kinetic configurations, the dynamical and evolutionary features. Some theoretical models only pay attention to the kinetic side, such as the velocity of bright front and the width of the bright region. As active phenomena, the dynamical features are important no less than those of the kinetic features, and should be studied in detail. The piston model could explain the major features of coronal transient on a large scale.

Observations show that the bright front of coronal transient moves with a constant velocity or only small acceleration, and the width of the bright region keeps increasing. In the piston model, the compression flow region ahead of the piston associates with the density increment relative to the quiet coronal density, and may be considered as the region of bright feature of the coronal transient. If the piston velocity is not too small, a shock wave is formed which is characterized by a larger density jump; and, in this case, the shock front ahead of the piston may 
be the observed bright front of the coronal transient, and the width between the piston and the shock front will be associated with the observed width of the bright region. The result of Figures 3 and 4 agree with the observed kinetic features of the transient: namely, the velocity of the front is nearly constant or accelerated but little, while the width keeps increasing. These conclusion depend on the velocity distribution of the ejected dense plasma, which is adopted here according to the typical trajectory of eruptive prominences. If the ejected dense plasma has different velocity distribution, so does the shock front; and, in this case, the bright front may be decelerating as suggested by a few examples of observed events.

On the dynamical side, the piston model could explain both the bright feature and the dark feature of the coronal transient at the same time. As the piston moves outward, there is a compressed flow region with higher plasma density ahead of the piston and a rarefaction region with lower plasma density behind the piston. According to the observations made with the coronagraph, the brightness is an indication of the density increment; and the darkness, of the density decrement. For the coronal transient associated with eruptive prominences, a bright region exists ahead of the prominence and a dark region behind the prominence; the bright and the dark regions may be explained as the compressed and the rarefaction regions, respectively. The plasma density in the bright region is generally one order of magnitude higher than the density in the quiet corona just ahead of the shock front as shown in Figures 5 and 6, and the density in the dark region is one order of magnitude lower than the density in the quiet corona just behind the level $R=R_{*}$ as shown in Figure 9. Therefore, both the bright features of the transient observed from the space coronagraph and the dark feature observed from the ground-based coronameter are explained by the piston model at the same time, and a global picture is completed. Furthermore, the density increment in the bright region and the density decrement in the dark region given by the piston model agree with those suggested by observations in their orders of magnitude. The piston model is different from the usual loop model in as much as it considers the gas-dynamical effects outside the loop, and the loop itself consists of a part of the piston.

On the evolutionary side, the observation suggest that the bright front is formed gradually - that is, there is no sharp front boundary at the beginning, as shown in Figure 2 of the previous paper ( $\mathrm{Hu}, 1983 \mathrm{a})$. The result of the present paper show that the shock wave is formed after a certain time, and the velocity and density change continuously from the values in the quiet corona to the values near the piston as given in Figures 5-8. Therefore, the bright region should be observed with a diffusion front in the beginning period, and then with a sharper front afterwards, and these features agree with the major evolutionary processes of the coronal transients.

The coronal transients have varied geometries in morphology, such as the loop, cloud, blob, ray, and streamer configurations. The ray and streamer coronal transients are explained by the dense plasma ejecting into a region with open 
configuration of magnetic field $(\mathrm{Hu}, 1983 \mathrm{c})$. The loop, cloud and blob transients are produced by the dense plasma ejecting into the region with closed magnetic field $(\mathrm{Hu}, 1983 \mathrm{a}, \mathrm{b})$. In the present paper, we discuss in detail the blob transient in the spherical coordinates, and similar mechanism may be applied to the loop transient in the cylindrical coordinates and will be analyzed elsewhere. The difference between the blob transient and the cloud transient is that the former has a sharp front boundary and the latter a diffusion front boundary. Figures 5 and 6 show that the total plasma density increment has different proportions contributed by shock jump and by the influence in the compressed region. The larger density increment by the shock jump may correspond to the configuration with sharp front, and the smaller density increment by the shock jump correspond to the diffusion boundary. Therefore, based on the results of the present paper, the larger velocity of the ejected dense plasma, the greater velocity and density increments at the shock front, and this may associate with the event which has sharp bright front such as the blob transient; otherwise, the smaller velocity of the ejected dense plasma, the weaker compression and smaller density increment of density at the shock front, and this may apply to the event with diffusion front such as the cloud transient. It will be interesting to compare these conclusions with the observations.

The energy source of the coronal transient is the most important problem for a theoretical model. The numerical models of computational tests often assume a pulsation of thermodynamical quantity or magnetic feidl being the driven source of the coronal transient. In contrast with that assumption, we consider that the kinetic energy of the eruptive prominence may be the driven force of the transient event $(\mathrm{Hu}, 1983 \mathrm{a})$. In principle, in the piston model the energy source of the event is the kinetic energy of the piston. which consists of the ejected dense plasma and the compressed magnetic region ahead of dense plasma. Both parts of the piston may contribute their kinetic energies to drive the coronal transient. The kinetic energy of the ejected dense plasma may be the major energy source of the transient if it is large enough, such as some events associated with the eruptive prominence $(\mathrm{Hu}, 1983 \mathrm{a})$; otherwise, the ejected dense plasma will change the configuration of the magnetic field and trigger off a large amount of kinetic energy in the compressed magnetic region. In this case, the Lorentz force in the magnetic region may be the major driven force. In the latter case, the mechanism is similar to the one of the loop models if the attention is paid only to the magnetic loop itself.

\section{Conclusions}

The piston model is different from the other theoretical models in its basic aspects. According to the above discussion, we can see that the piston model of the coronal transient give not only the global features of transient, but also the evolutionary processes, and the theoretical results agree with the observations in kinetic, dynamical and also the morphologic features. The major observation phenomena are reasonably explained. 
From the viewpoint of gas dynamics, the applied gravity is important in the processes of coronal transient; and we should study the piston problem in the gravitational field, which is different obviously from the usual piston problem. In comparison with the usual piston problem in gas dynamics, the plasma velocity in the compression flow region is larger and the shock strength is weaker; therefore, the plasma density increment in the compressed region in the case including the applied gravity is larger than the one in the case excluding the applied gravity. These conclusions are useful in the applications of the theoretical works. It will be interesting to study the piston model in detail further, such as the evolution of the piston structure, the unsteady two-dimensional picture of piston model and others.

In the present paper, we discuss that the dense plasma ejects once into the interplanetary space. However, before a major ejection of dense plasma, a small amount of mass may be ejected with energy, or the dense plasma moves slowly first and then accelerates. In these cases - proceeding the major plasma density increment - there is a small and gradual density increment, which may be corresponding to the structure of the forerunner of transient observed by the space coronagraph (Jackson and Hildner, 1978). Similar analysis may be applied to discuss this phenomenon on the coronal transient events, and we will study it in detail elsewhere.

\section{References}

Allen, C. W.: 1973, Astrophysical Quantities, Athlone Press, London, p. 220.

Anzer, U.: 1978, Solar Phys. 57, 111.

Anzer, U. and Pneuman, G. W.: 1982, Solar Phys. 79, 129.

Dryer, M.: 1982, Space Sci. Rev. 33, 233.

Dryer, M., Wu, S. T., Steinolfson, R. S., and Wilson, R. M.: 1979, Astrophys. J. 227, 1059.

Hu, W. R.: 1983a, Astrophys. Space Sci. 92, 373.

Hu, W. R.: 1983b, Astrophys. Space Sci. 92, 395.

Hu, W. R.: 1983c, Acta Astron. Sinica 24, 401.

Hu, W. R.: 1984, Appl. Math. Mech. (in press)

Jackson, B.V. and Hildner, E.: 1978, Solar Phys. 60, 155.

Liu, X. P.: 1983, Acta Astrophys. Sinica 3, 23.

MacQeen, R. M.: 1980, Phil. Trans. Roy. Soc. London A297, 605.

Mouschovias, T. and Poland, A. I.: 1978, Astrophys. J. 220, 675.

Munro, R. H., Gosling, J. T., Hildner, E., MacQeen, R. W., Poland, A. I., and Ross, C. L.: 1979, Solar Phys. 61, 201.

Nakagawa, Y., Wu, S. T., and Han, S. M.: 1978, Astrophys. J. 219, 314.

Nakagawa, Y., Wu, S. T., and Han, S. M.: 1981, Astrophys. J. 244, 331.

Pneuman, G. W.: 1980, Solar Phys. 65, 369.

Rust, D. M. Hildner, E., Dryer, M., Hansen, R. T., McClymont, A. N., McKenna-Lawlor, S. M. P., McLean, D. J., Schmahl, E., Steinolfson, R. S., Tandberg-Hanssen, E., Tousey, R., Webb, D., and Wu, S. T.: 1979, in P. Sturrock (ed.), Solar Flares, University of Colorado Press, p. 273.

Steinolfson, R. S. and Nakagawa, Y.: 1977, Astrophys. J. 215, 345.

Tang, Z. M. : 1984a, Acta Mech. Sinica, (in press).

Tang, Z. M.: 1984b, Acta Mech. Sinica, (in press).

Wu, S. T., Dryer, M., Nakagawa, Y., and Han, S. M.: 1978, Astrophys. J. 219, 325.

Yeh, T.: 1982, Solar Phys. 78, 287.

Yeh, T. and Dryer, M.: 1981, Astrophys. J. 245, 704. 